Gut and Liver, Vol. 13, No. 4, July 2019, pp. 383-384

\title{
Nonalcoholic Fatty Liver Disease Is a Stepping Stone in the Path toward Diabetes Mellitus
}

\author{
Nae-Yun Heo \\ Department of Internal Medicine, Inje University Haeundae Paik Hospital, Inje University College of Medicine, Busan, Korea
}

See "Improvement of Nonalcoholic Fatty Liver Disease Reduces the Risk of Type 2 Diabetes Mellitus" by Hyo Jung Cho, et al. on page 440, Vol. 13, No. 4, 2019

It has been a long-standing dogma that nonalcoholic fatty liver disease (NAFLD) is a hepatic manifestation of metabolic syndrome, in which diabetes mellitus (DM) is one of the primary components. A few meta-analyses associated NAFLD with the risk of incidental DM, and patients with more severe NAFLD were likely to develop incident DM. ${ }^{1,2}$ However, few studies explored whether the changes in NAFLD over a period of time influenced the risk of incident DM. In this issue of Gut and Liver, Cho et al. ${ }^{3}$ presented two pertinent results. First, incident and persistent NAFLD were independent risk factors for predicting DM incidence. Second, more notably, the risk of incident DM did not increase in patients with resolved NAFLD compared with NAFLD-naïve patients during a median 5-year follow-up. This finding is important because if the risk of incident DM was reduced by the improvement of NAFLD, the treatments targeting NAFLD may be a stepping stone on the path to elimination of new-onset DM in patients with high cardiovascular risk.

According to previous studies, Sung et al. ${ }^{4}$ reported that newonset fatty liver disease was associated with incident DM, and the progression of fatty liver was related to a marked increase in the risk of incident DM based on the baseline and 5-year data. Yamazaki et al. ${ }^{5}$ showed that type 2 DM occurred in 6.4\% and $17.8 \%$ of patients with improved and sustained NAFLD over 10 years, respectively. Also, they insisted that amelioration of NAFLD was associated with reduced incidence of type 2 DM, based on a logistic regression analysis. Although these studies reported by Sung et al. ${ }^{4}$ and Yamazaki et al. ${ }^{5}$ suggested that the changes of fatty liver altered the risk of DM occurrence, they incorporated the time-dependent variable (change of fatty liver) into the logistic regression, and if they failed to adopt the specific statistical approach such as the generalized methods of moments or the generalized estimating equation, the estimated odds ratio may be less accurate. However, this scenario was not discussed clearly. In contrast, Cho et al. ${ }^{3}$ performed the Cox proportional-hazards regression with survival data derived from baseline until DM occurrence, and apparently suggested that the progression and regression of NAFLD were linked to positive and negative risk of incident DM, respectively. In this context, the authors mentioned that their study was the first of its kind to confirm a causal relationship between the dynamic changes of NAFLD and time to DM occurrence.

Nevertheless, the possibility of unadjusted confounding factors associated with the independent and dependent variables, cannot be excluded. The authors included the changes in obesity status as a covariate in multivariable Cox regression. However, this variable is not an ordinal or interval scale but represents a nominal scale. Therefore, changes in body weight may still be a potential confounder. Also, if the authors intended to evaluate the impact of changes in fatty liver among the population with similar characteristics, it would be better to compare the nonNAFLD versus incident NAFLD group (NAFLD is absent at baseline), and the persistent and resolved NAFLD group (NAFLD is present at baseline), separately. Nonetheless, we can appreciate the considerable difference in the risk of incident DM between the non-NAFLD versus incident NAFLD group and the persistent and resolved NAFLD group based on Kaplan-Meier curves, which is the most impressive feature of this study.

It is important to note that this impact of fatty liver change on the risk of DM was observed not only in obese patients but also in nonobese patients at baseline in this study. Because

Correspondence to: Nae-Yun Heo

Department of Internal Medicine, Inje University Haeundae Paik Hospital, Inje University College of Medicine, 875 Haeun-daero, Haeundae-gu, Busan 48108, Korea

Tel: +82-51-797-2446, Fax: +82-51-797-1340, E-mail: nyheo@hanmail.net

pISSN 1976-2283 eISSN 2005-1212 https://doi.org/10.5009/gnl19192

@) This is an Open Access article distributed under the terms of the Creative Commons Attribution Non-Commercial License (http://creativecommons.org/licenses/by-nc/4.0) which permits unrestricted non-commercial use, distribution, and reproduction in any medium, provided the original work is properly cited. 
weight gain even within the range of nonobese body mass index is associated with the development of nonobese NAFLD, weight loss may lead to remission of nonobese NAFLD, and reduce the risk of incident DM eventually. ${ }^{6}$ This observation is useful in managing nonobese NAFLD patients in the clinic.

Despite evidence suggesting the impact of fatty changes on the onset of DM, the underlying biological mechanism has yet to be elucidated. The pathogenesis of DM in NAFLD patients may be mediated via several hepatokines such as Fetuin-B associated with impaired metabolic control. In addition, in the case of fatty liver, lipotoxicity of diacylglycerol may hamper the insulin signaling, and thereby promote insulin resistance. ${ }^{7}$ We expect that the studies investigating the mechanisms ranging from NAFLD to DM will elucidate the potential therapeutic targets in the future.

In conclusion, the study by Cho et al. ${ }^{3}$ supports the hypothesis that NAFLD is not only a phenomenon of metabolic syndrome, but also a modifiable trigger factor associated with progression to the advanced stages of insulin intolerance, or DM. Thus, we have another reason to manage NAFLD for the prevention of catastrophic events.

\section{CONFLICTS OF INTEREST}

No potential conflict of interest relevant to this article was reported.

\section{ORCID}

Nae-Yun Heo

https://orcid.org/0000-0001-6571-8935

\section{REFERENCES}

1. Ballestri S, Zona S, Targher G, et al. Nonalcoholic fatty liver disease is associated with an almost twofold increased risk of incident type 2 diabetes and metabolic syndrome: evidence from a systematic review and meta-analysis. J Gastroenterol Hepatol 2016;31:936-944.

2. Mantovani A, Byrne CD, Bonora E, Targher G. Nonalcoholic fatty liver disease and risk of incident type 2 diabetes: a meta-analysis. Diabetes Care 2018;41:372-382.

3. Cho HJ, Hwang S, Park JI, et al. Improvement of nonalcoholic fatty liver disease reduces the risk of type 2 diabetes mellitus. Gut Liver 2019;13:440-449.

4. Sung KC, Wild SH, Byrne CD. Resolution of fatty liver and risk of incident diabetes. J Clin Endocrinol Metab 2013;98:3637-3643.

5. Yamazaki H, Tsuboya T, Tsuji K, Dohke M, Maguchi H. Independent association between improvement of nonalcoholic fatty liver disease and reduced incidence of type 2 diabetes. Diabetes Care 2015;38:1673-1679.

6. Kim D, Kim WR. Nonobese fatty liver disease. Clin Gastroenterol Hepatol 2017;15:474-485.

7. Lonardo A, Lugari S, Ballestri S, Nascimbeni F, Baldelli E, Maurantonio M. A round trip from nonalcoholic fatty liver disease to diabetes: molecular targets to the rescue? Acta Diabetol 2019;56:385396. 